\title{
Contrast radiography of the nasopharynx
}

\author{
GORDON EVISON \\ M.B.Ch.B., D.M.R.D., F.F.R. \\ Department of Diagnostic Radiology, \\ United Bristol Hospitals*
}

\section{Summary}

A technique of contrast radiography of the nasopharynx is described and indications for the procedure are reviewed. The main advantage of using this method is the simple and effective way it outlines the tumour mass and shows its response to therapy and any localized recurrences. Characteristic features of tumours at different sites are illustrated. The potential usefulness of this technique in detecting early lesions is emphasized.

\section{Introduction}

Carcinoma of the nasopharynx is often diagnosed only late and with difficulty. Mortality is high, and the results of treatment have hardly improved over the past two decades. Because of its inaccessibility, indirect methods of examining the nasopharynx, such as radiography, would seem to be specially valuable. However, plain films of the nasopharynx, while essential for demonstrating bone destruction by the tumour if this is present, do not always clearly define the soft-tissue mass. Attempts to use positive contrast media in the nasopharynx were first made in 1934 by Ruëdi \& Zuppinger, using Lipiodol. More recently barium has been used (Lederman, 1961 ; Khoo, Chia \& Nalpon, 1967a ; Khoo, Kanagrasuntheram \& Chia, 1967b), and oily Dionosil (Jing \& McGraw, 1965).

The following is a description of the technique used at the Bristol General Hospital, with a review of the normal anatomy of the contrastfilled nasopharynx, the indications for its use, and an assessment of its present usefulness as a diagnostic procedure.

\section{Technique}

No preliminary preparation of the patient is required. With the patient supine, a submentovertical (SMV) plain radiograph is taken on a $12 \times 10$ in. film, using the Schonander head unit. A horizontal-beam lateral plain radiograph is also taken with the patient's head in SMV posi-

\footnotetext{
*Present address: Royal United Hospital, Bath.
}

tion, on a $12 \times 10$ in. film. Each nasal cavity is then sprayed with $4 \%$ lignocaine local anaesthetic. A soft rubber catheter is introduced through the anterior naris on one side and part way along the inferior meatus, with the head in the SMV position. A syringe containing $20 \mathrm{ml}$ aqueous Dionosil is attached to this and the injection begun (Fig. 1). It is not necessary to have the tip of the catheter in the nasopharynx, as gravity will take the medium there; neither is it necessary to inject both sides unless one side is blocked for some reason. The patient breathes through his mouth and is

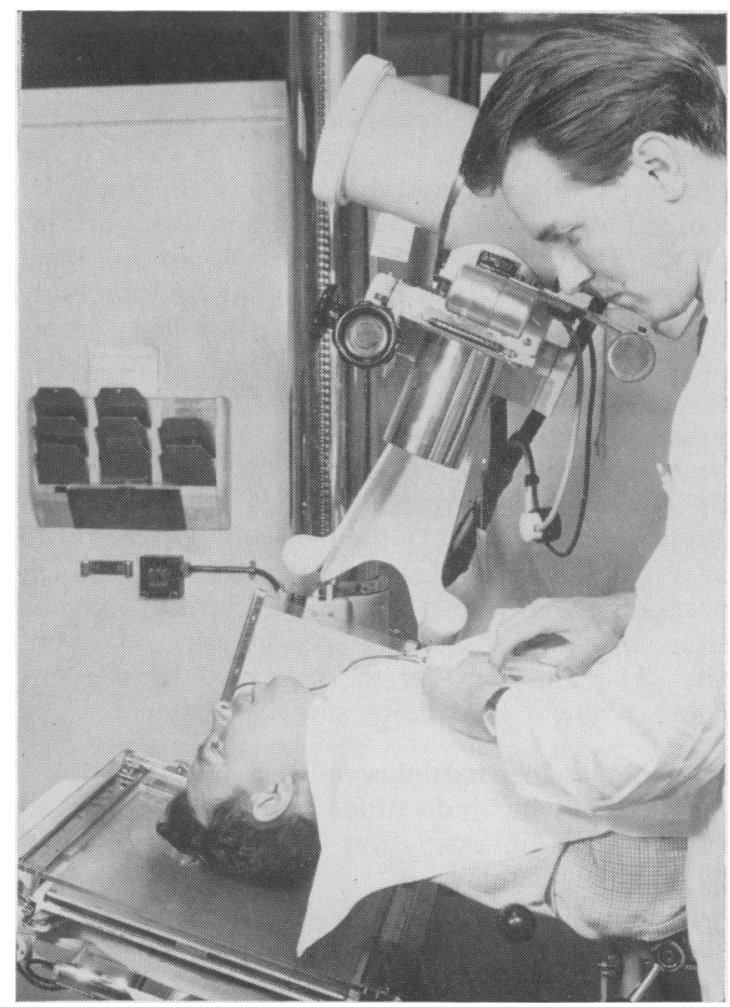

FIG. 1. Introduction of contrast medium into the nasopharynx. 
instructed not to swallow. When the patient feels the urge to swallow (but without swallowing), he gives a pre-arranged signal and the injection is stopped. Usually $8-15 \mathrm{ml}$ are necessary and at this point the nasopharynx is full of contrast medium. Twelve $\times 10$ in. SMV and horizontal beam lateral SMV radiographs are then exposed as rapidly as possible. If it is desired to fill the Eustachian tubes, the patient then swallows, with his head still in the SMV position, and a further $12 \times 10$ in. SMV radiograph is exposed. The Valsalva manoeuvre may be done to reinforce this, though it has been found that it has no added advantage over simple swallowing and is much more difficult for the patient. The contrast medium is then blown out of the nose into paper handkerchiefs by the patient, or swallowed aided by a glass of fruit juice. The essence of obtaining satisfactory radiographs is to fully explain to the patient what is about to happen before starting, and if this is done then the procedure is very well tolerated, even by small children.

\section{Normal anatomy}

On the SMV view, the contrast-filled nasopharynx appears somewhat dumb-bell shaped and normally quite symmetrical. The nasal septum separates the two nasal cavities, and the inferior turbinates usually show as filling defects within these. The pharyngeal orifices of the Eustachian tubes outline, bounded posteriorly by the torus tubarius and behind this the fossa of Rosenmüller (Fig. 2) The cartilaginous portions of the Eustachian tubes fill for about an inch, but we have never observed filling of the bony tube or middle ear, though this has been recorded (Wittenborg \& Neuhauser, 1963). The lateral recesses of the nasopharynx are subject to considerable individual variations, and these have been studied using positive contrast media and ciné fluorography by Khoo et al. (1967a, b). On the lateral view (Fig. 3), the roof and posterior wall of the nasopharynx are usually smooth, though occasionally in adults small irregularities may be seen as a normal feature due to adenoid remnants. The anterior margin is formed by the soft palate, which is usually clearly outlined.

\section{Indications for nasopharyngography}

The following indications have been advanced for contrast investigations of the nasopharynx by various authors:

(1) When a nasopharyngeal growth has been detected clinically, or on a plain film, and it is desired.

(a) to see the extent and size of the growth,

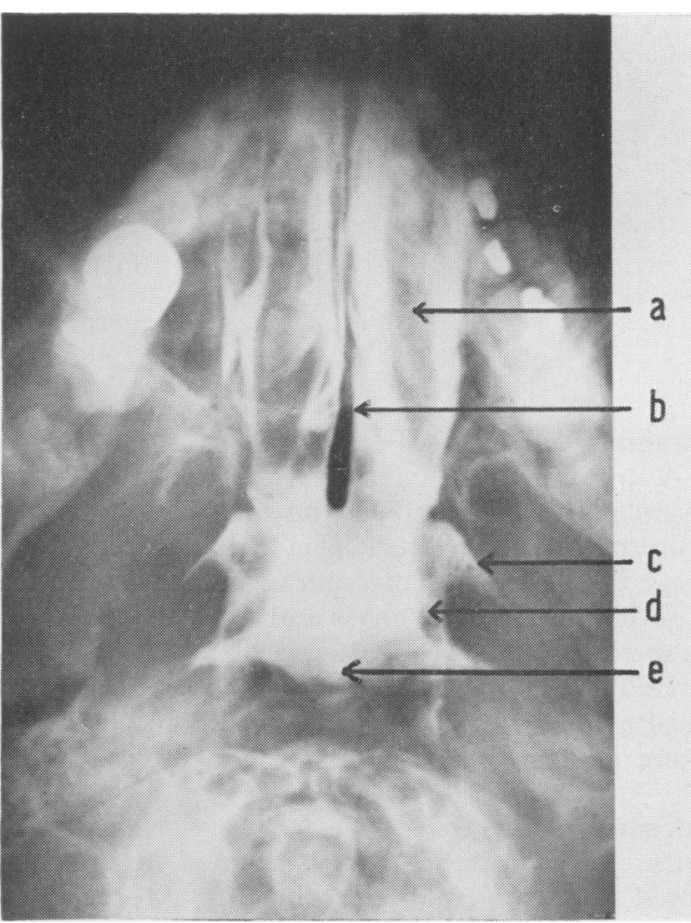

FIG. 2. Normal nasopharyngogram-submento-vertical. Note the bilateral symmetry, and the partial filling of the Eustachian tubes. (a) Inferior turbinate, (b) Nasal septum, (c) pharyngeal orifice of the Eustachian tube, (d) torus tubarius and fossa of Rosenmüller, (e) posterior wall of the nasopharynx.

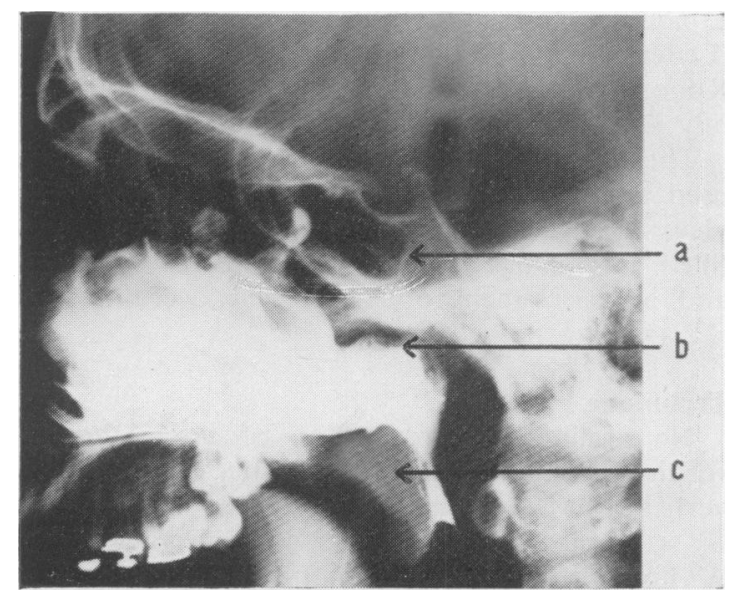

FIG. 3. Normal nasopharyngogram-lateral view. (a) Sphenoid sinus, (b) postero-superior wall of the nasopharynx, (c) soft palate. 
(b) to see if the Eustachian tubes are affected.

(2) Where a nasopharyngeal growth is still suspected clinically, though plain films and endoscopy have failed to locate it.

(3) Any case of malignant enlargement of cervical nodes where a primary is being sought.

(4) To show the response of nasopharyngeal neoplastic masses to treatment, e.g. radiotherapy or surgery.

(5) Occasionally, to demonstrate the patency of the Eustachian tubes prior to operations such as tympanoplasty.

(6) Occasionally, to show other lesions which may not be fully visible on endoscopy, e.g. hypertrophied posterior turbinates, choanal polypi, choanal atresia.

\section{Nasopharyngeal tumours}

These may arise in the following sites:

(1) The lateral wall, either the fossa of Rosenmüller or the region of the torus tubarius.

(2) The postero-superior wall or roof.

(3) The nasopharyngeal surface of the soft palate.

(4) Undetermined site of origin.

(5) Extrinsic tumours invading the nasopharynx.

(1) Tumours arising from the lateral wallthis is the most common site of origin, usually from one or other fossa of Rosenmüller. They are shown best on the submento-vertical projection, appearing as an indentation or filling defect on the lateral margin of the contrast medium, obliterating the fossa of Rosenmüller or torus tubarius. On the lateral view filling defects are usually shown on the postero-superior margin of the contrast medium. Fig. 4 shows a large anaplastic carcinoma arising from the left lateral wall.

(2) Tumours arising from the postero-superior wall or roof-these show as a soft tissue mass projecting into the cavity of the nasopharynx and causing filling defects on the postero-superior margins of the contrast medium as shown on the lateral view. If the tumour is small, the submento-vertical view may appear normal, but with larger tumours deficient filling of the posterior margin of the contrast medium is usually evident, often more on one side than the other. Fig. 5(a) and (b) shows a large tumour arising from the postero-superior wall and almost completely occluding the nasopharynx.

(3) Tumours arising from the nasopharyngeal surface of the soft palate-these show as an increase in thickness of the soft palate, seen best on the lateral view (Fig. 6).

(4) Tumours whose site of origin cannot be determined-the larger tumours may fill the whole of the nasopharynx and obstruct the passage of contrast beyond the posterior choanae. In these circumstances the exact site of origin cannot be determined.

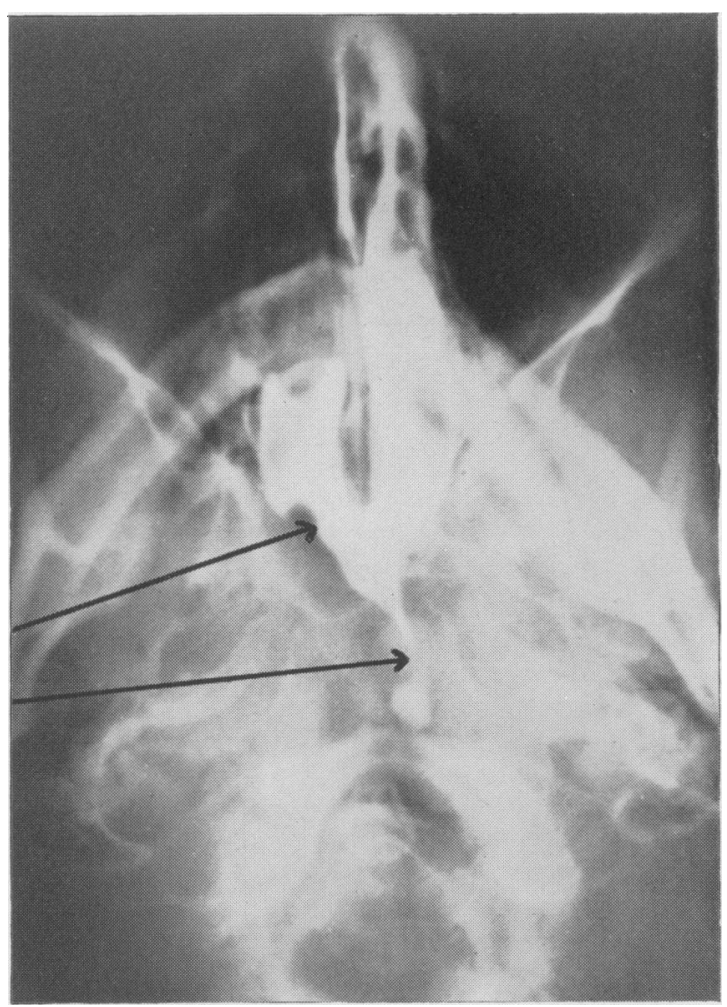

FIG. 4. Anaplastic carcinoma (arrow) arising from the left lateral wall, causing a large filling defect in the contrast medium. (Reproduced by kind permission of the Editor of J. Laryng.)

(5) Tumours arising in surrounding structures may invade the nasopharynx. Fig. 7(a) and (b) shows a large cylindroma which had spread from the left maxillary antrum into the nasal cavity and nasopharynx.

\section{Discussion}

The technique of positive contrast radiography of the nasopharynx described here is simple to perform, requires only standard radiological equipment and does not cause undue discomfort to the patient. The main value at the present time of using this method of investigation is the clear demonstration it provides of the site of origin 



Fig. 5. Tumour (arrows) arising from the posterosuperior wall, almost completely occluding the nasopharynx. (a) Submento-vertical view. (b) Lateral view. (Reproduced by kind permission of the Editor of J. Laryng.)

and extent of nasopharyngeal tumours, and in showing their response to radiotherapy (Pearl-

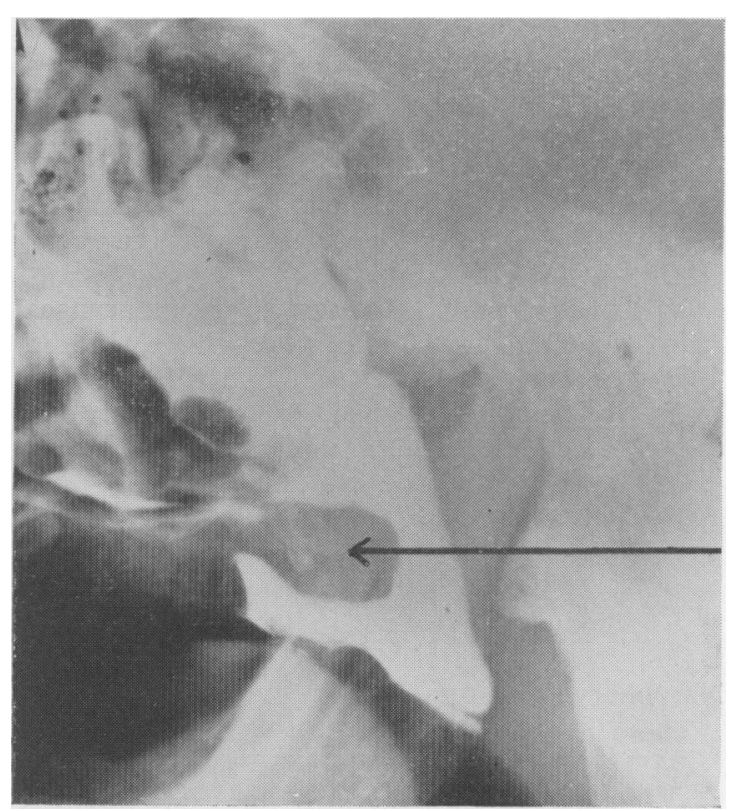

FIG. 6. Carcinoma (arrow) arising from the nasopharyngeal surface of the soft palate.

man, 1961 ; Morgan \& Evison, 1966; Evisonฉ 1967). It is also valuable for showing localize tumour recurrences (Edland, 1967). The most ims portant problem with these tumours is early $\overrightarrow{0}$ recognition, and the only way of increasing ther. number of survivors is to diagnose and treat the neoplasm at a much earlier stage (Lancet, 1966). Vilar (1966) found that the average time between the onset of symptoms and a definite diagnosis was $9 \cdot 7$ months, and in three of his twenty-four cases direct examination of the post-nasal space and biopsy were at first negative. Most of our cases were referred when the lesion was well advanced, but in one case which presented with enlarged cervical glands and in whom endoscopy failed to reveal a lesion, a nasopharyngogram (Fig. 8) showed a shallow filling defect on the right side which on biopsy proved to be an anaplastic carcinoma. Detection of small lesions is necessarily difficult, but the procedure does offer a way of diagnosing at least some of these at a much earlier stage than at present. It should be considered whenever clinical suspicion of a nasopharyngeal tumour arises.

\section{Acknowledgments}

I am grateful to Professor J. H. Middlemiss and Dr E. Rhys Davies for their help and advice and to the Consultant E.N.T. and Radiotherapy staff of the United Bristol Hospitals for referral of cases, to Mr J. E. Hancock for the reproductions and to Miss A. Sainsbury for the typescript. 

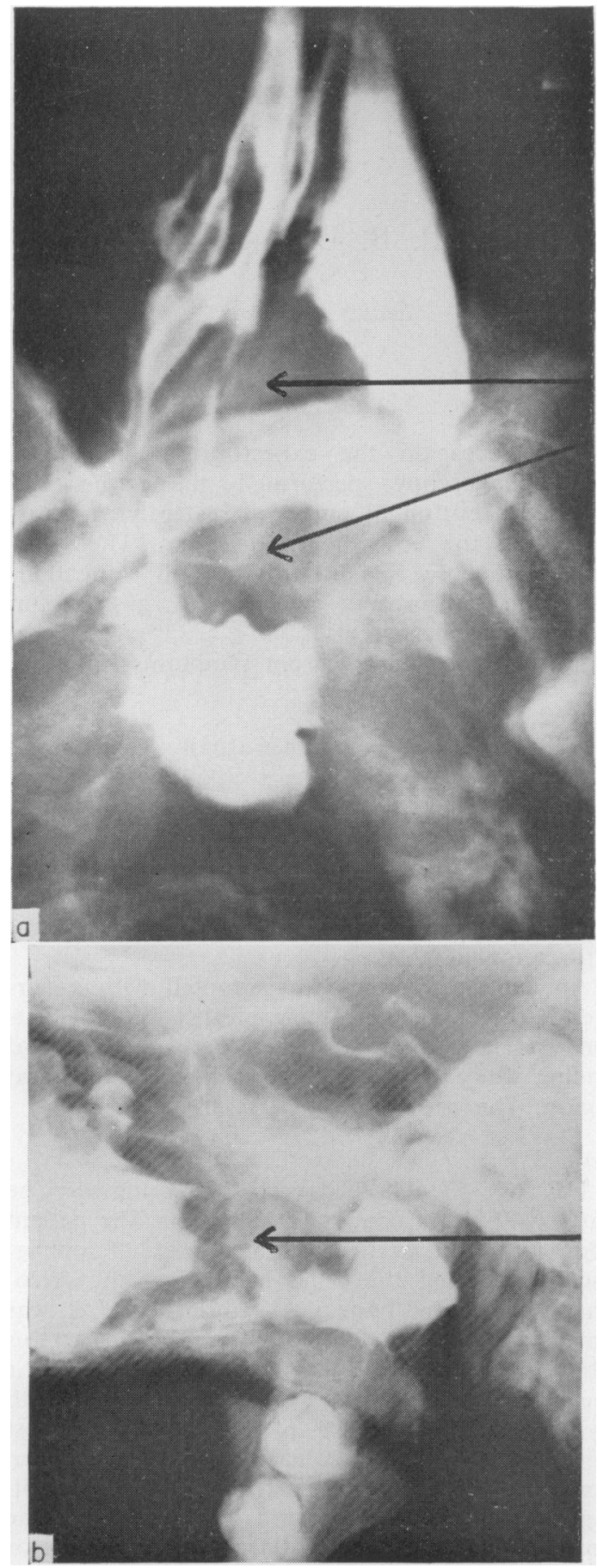

FIG. 7. Cylindroma (arrows) arising from the left maxillary antrum and invading the nasal cavity and nasopharynx. (a) Submentovertical view. (b) Lateral view.

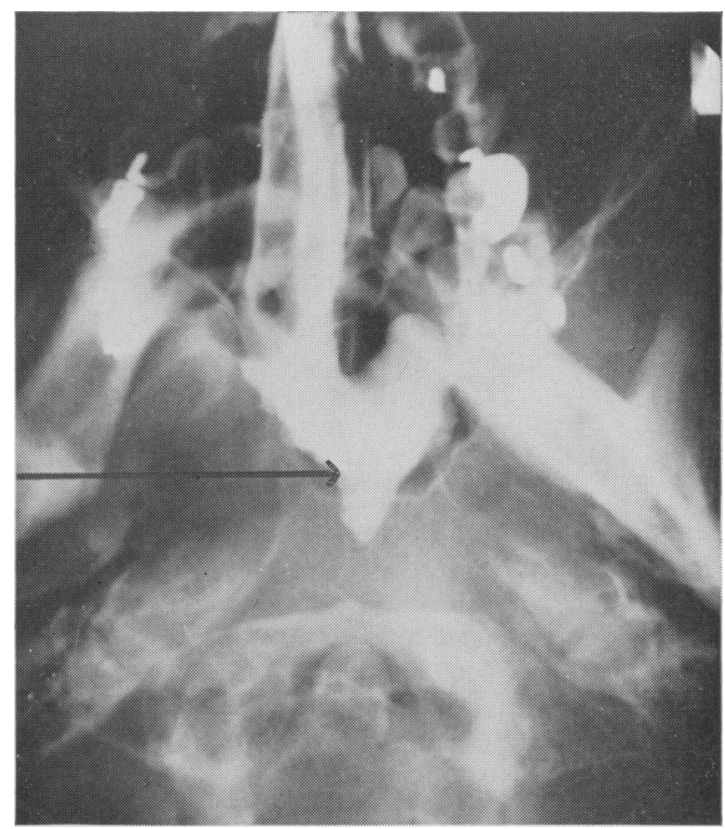

FIG. 8. Early anaplastic carcinoma (arrow) arising from the right lateral wall, not visible on endoscopy.

\section{References}

EDLAND, R.W. (1967) Nasopharyngeal carcinoma-a simplified technique of individualized intracavity therapy for superficially recurrent or persistent disease in the conscious patient. Radiology, 89, 341.

Evison, G. (1967) Carcinoma of the nasopharynx. Lancet, i, 157.

JiNG, B.S. \& McGRaW, J.P. (1965) Contrast nasopharyngography in the diagnosis of tumours. Arch. Otolaryng. 81, 365 .

Khoo, F.Y., Chia, K.B. \& NalPon, J. (1967a) A new technique of contrast examination of the nasopharynx with ciné fluorography and roentgenography. Amer. J. Roentgenol. 99, 238.

Khoo, F. Y., Kanagasuntheram, R. \& Chia, K.B. (1967b) Variations of the lateral recesses of the nasopharynx. Arch. Otolaryng. 86, 456.

Lancet (1966) Annotation, ii, 1455.

LEDERMAN, M. (1961) Cancer of the Nasopharynx, its Natural History and Treatment, p. 18. Thomas, Springfield, Illinois.

MoRgan, N.V. \& Evison, G. (1966) Contrast radiography in the follow-up of nasopharyngeal tumours. J. Laryng. 80, 699.

Pearlman, A.W. (1961) Serial radiography to assist irradiation of carcinoma of the nasopharynx. Radiology, 77, 543.

RÜEDI, L. \& ZUPPINGER, A.u. (1934) Zur roentgenkontrastuntersuchung der Nasopharynx. Hals-, Nas.-Ohrenbeilk. 35, 500 .

VILAR, P. (1966) Nasopharyngeal carcinoma-a report on 24 patients seen over six years. Scott. med. J. ii, 315.

Wittenborg, M.H. \& Neuhauser, E.B.D. (1963) Simple roentgenographic demonstration of eustachian tubes and abnormalities. Amer. J. Roentgenol. 89, 1194. 